\title{
Neural Gliding Techniques for the Treatment of Carpal Tunnel Syndrome: A Systematic Review
}

\author{
Jennifer M. Medina McKeon and Kathleen E. Yancosek
}

\begin{abstract}
Context: There are discrepancies regarding the efficacy of neural gliding exercises for the management of carpal tunnel syndrome (CTS). Objective: To conduct a systematic review assessing the efficacy of neural gliding in comparison to alternative nonsurgical treatment for the management of CTS. Evidence Acquisition: A computerized search was performed in April 2008. Criteria for inclusion required that studies (1) were written in English, (2) examined the efficacy of neural gliding techniques for treatment of CTS, and (3) included at least one of the selected patient-oriented outcomes. Effect sizes, relative risk, and 95\% confidence intervals were calculated to compare neural gliding to alternative treatment. Evidence Synthesis: Six studies met inclusion criteria. For all variables, none were consistently favorable toward neural gliding over alternative treatment. However, comparisons across studies revealed a possible trend toward improved outcomes with the use neural gliding. Conclusions: The efficacy of neural gliding is not clear. More research is necessary to determine the population that may respond optimally to this treatment. Keywords: mobilization, decompression, wrist
\end{abstract}

\section{Context}

Carpal tunnel syndrome (CTS) is the most common peripheral neuropathy. ${ }^{1}$ It accounts for 2 billion U.S. dollars in surgical costs each year, ${ }^{2}$ and those suffering from CTS have the highest reported rate of work days missed. ${ }^{3}$ In 1998, 3 out of every 10 workers lost work due to CTS. Half of the workers missed more than 10 days of work. ${ }^{4}$ Patients with CTS typically reported symptoms of numbness, tingling, and nocturnal burning pain. Clinical signs may include a decrease in both discriminative and light touch sensation, and in advanced cases, a loss of grip and pinch strength. ${ }^{1}$

CTS has also been cited as one of the most common neuropathies in sports. ${ }^{5-9}$ Significant increases in carpal tunnel pressure have been observed in patients performing wrist flexion and extension, making a fist, holding an object, and

The authors are with the University of Kentucky Rehabilitation Sciences Department in Lexington. E-mail: jennifer.medina@uky.edu. 
isometric finger flexion against resistance. ${ }^{10}$ Athletes participating in sports that require such actions report a higher prevalence of CTS than sports that do not. Cycling, ${ }^{9,11}$ football, ${ }^{8}$ golf, ${ }^{9,12}$ hockey, ${ }^{5}$ lacrosse, ${ }^{5}$ rock climbing, ${ }^{13}$ weightlifting, ${ }^{8}$ wheelchair athletics, ${ }^{9,14}$ and wrestling ${ }^{8}$ are all sports that have been implicated as those with increased risk of CTS.

CTS is thought to be caused by compression on the median nerve resulting in ischemia or mechanical injury. ${ }^{15}$ This condition may result from space-occupying lesions within the tunnel, such as cysts, tumors, osteophytes, fracture callus, or hypertrophic synovial tissue. It has also been tied to metabolic or systemic conditions such as thyroid disease, diabetes mellitus, rheumatoid arthritis, alcoholism, and pregnancy. ${ }^{16}$ In addition to compression and systematic disease, CTS has also attributed to a decrease in longitudinal excursion of the median nerve as it passes through the carpal tunnel. ${ }^{17-22,23}$

Neural gliding is a treatment technique that may improve symptoms related to CTS. There is evidence that median nerve excursion can be influenced by neural gliding techniques, as demonstrated in a cadaveric study. ${ }^{24}$ Many conservative and post-operative interventions for the management of CTS include neural gliding exercises in order to improve nerve excursion. Sometimes referred to as "neural flossing" or "neurodynamic mobilization," the technique of neural gliding is movement based and attempts to take the nerve throughout the available range of motion, potentially affecting the nerve both mechanically and physiologically. ${ }^{23,24}$ Neural gliding may improve the actual excursion of the nerve, decreasing adhesions and reducing symptoms by allowing the nerve to move freely. This technique may also help to oxygenate the nerve, decreasing ischemic pain. Though these exercises are commonly prescribed, there has been little research performed to support their use and justify their clinical value.

Three previous systematic reviews ${ }^{25-27}$ that included neural gliding were identified. Two systematic reviews were performed on the same two studies; ${ }^{28,29}$ however, differing conclusions were drawn. O'Conner et $\mathrm{al}^{27}$ concluded there were no significant benefits to neural gliding, whereas Muller et $\mathrm{al}^{26}$ recommended neural gliding for the benefit of reduced pain. A third systematic review by GoodyearSmith and Arroll $^{25}$ concluded that there was possible benefit in reduced rates of surgical intervention with the use of neural gliding. In evaluation of these three systematic reviews, we identified that there were additional research studies that examined the efficacy of nerve gliding exercises in the treatment of CTS that were not included in these previous reviews. We also recognized that these previous reviews offered few recommendations for future research in this area. The lack of converging conclusions of the three previous systematic reviews, as well as the addition of more current information to the scientific literature prompted the current systematic review.

\section{Objective}

The purpose of this systematic review was to answer the following clinical question: Does neural gliding enhance patient outcomes associated with CTS when compared to alternative nonsurgical treatment? We also intended to identify specific areas for future research of neural gliding exercises for the treatment of CTS. 


\section{Evidence Acquisition}

Eight patient-oriented outcome measures were specifically chosen to reflect current clinical practice standards. Studies included in this sytematic review reported measures for at least one of the selected outcomes. Two studies provided outcomes for all eight measures.

\section{Self-Reported Outcomes}

Pain. Pain was scored using the Visual Analog Scale $(\mathrm{cm})$. This has been determined to be a valid, reliable, and responsive clinical measure of pain. ${ }^{30-34}$

Symptoms (SSS). Severity of symptoms was documented with the use of the Symptom Severity Scale (SSS). With the SSS, patients are asked to rate the severity of their symptoms related to CTS. The SSS has been determined valid, reliable, and responsive for the use in this population..$^{35}$

Self-Reported Function. Self-reported function was measured with the use of the Functional Severity Scale (FSS). Patients rate the difficulty with which they were able to perform daily tasks. The FSS is valid, reliable, and responsive for the use of determining perceived functional status. ${ }^{35}$ Self-reported function was also tested using the Box Test, for which the patient scores perceived ability to perform tasks.

\section{Clinical Examination}

Phalen's Test and Tinel's Test. Phalen's test is a clinical special test that evaluates the recreation of symptoms with passive compression of the median nerve with the wrist in hyperflexion and hyperextension. ${ }^{36}$ With Tinel's test, the clinician attempts to recreate the symptoms of a neural pathology by tapping a superficial aspect of the nerve over the carpal tunnel. ${ }^{36}$ These tests are a commonly used clinically for the detection of nerve pathologies.

\section{Two-Point Discrimination}

A measure of sensory acuity and light touch, two-point discrimination is tested by measuring the smallest distance a patient can perceive two pinpricks as separate units. This is a commonly used measure to determine the degree to which deficits exist in the sensory function of the median nerve. ${ }^{37}$

\section{Strength}

Grip Strength and Pinch Strength. Grip and pinch strength were evaluated with the use of dynamometry. Grip strength has been used as a functional assessment of the upper limb ${ }^{38}$ and has been shown to be valid and reliable even among people with different grip spans. ${ }^{39,40}$ Pinch strength is a measure of the strength and coordination of the intrinsic and extrinsic muscles of the hand. It is also commonly used to assess motor function in patients with CTS. 
For the outcomes of the VAS, SSS, self-reported function, and 2-point discrimination, a reduction in score indicates improvement. For measures of grip strength and pinch strength, an increase in outcome values indicates improvement.

\section{Study Search and Selection}

In April 2008, investigators performed a computerized literature search of PubMed Central (to April 2008, CINAHL; 1982 - April 2008), Medline (1980 - April 2008), the Cochrane Central Register of Controlled Trials (1964 - April 2008), and SPORTDiscus (1980 - April 2008) to identify citations concerning the efficacy of neural gliding exercises for the nonsurgical management of CTS. Searches included the key terms "nerve glides," "nerve gliding exercises," "nerve mobilization exercises," "upper limb neuropathy," "therapy," "entrapment neuropathy," "nerve gliding exercises" and "carpal tunnel decompression exercises."

Searches were limited to "human" and "English" studies reported in peerreviewed journals. A hand search for relevant citations was also performed on all retrieved studies. Studies were selected independently by two investigators (JM and KY). Studies were eligible for inclusion if they addressed neural gliding exercises for the treatment of CTS, included a comparison treatment group, and utilized at least one of the eight selected outcomes measures. To perform statistical analysis, it was necessary that the included studies provided means and standard deviations for all non-dichotomous outcomes.

\section{Assessment of Methodological Quality}

The methodological quality of each study was assessed independently by the investigators using the Physiological Evidence Database (PEDro) rating scale. Two researchers rated all studies independently. In the event of a conflict, a third party was consulted to determine the score.

\section{Data Extraction}

Means and standard deviations for both neural gliding and comparison groups were obtained for all continuous outcome measures. Certain studies ${ }^{29,41}$ made more than one comparison of neural gliding techniques with alterative nonsurgical treatments. These were treated as separate studies, as they were comparing different groups to the neural gliding exercises. Counts of success and failures were obtained for all dichotomous variables.

\section{Statistical Analysis}

For the continuous variables (pain, symptoms, self-reported function, 2-pt discrimination, grip strength, and pinch strength), effect sizes and 95\% confidence intervals were calculated to determine the magnitude of the effect between the neural gliding groups and the comparison groups (true control or alternate therapy). Effect sizes were interpreted as $<.4$ is small, .4 to .7 moderate, and $>.7$ is large. ${ }^{42}$ For the dichotomous variables (Phalen's test and Tinel's test), relative risk and 
95\% confidence intervals were calculated, along with the risk difference. Metaanalysis was not performed due to the heterogeneity of study design related to neural gliding exercise prescription, follow-up periods, and comparison (control) group courses of treatment. Effect sizes, relative risk, and risk differences were calculated in MATLAB (MATLAB R2007b, The Mathworks, Inc., Natick, MA). One investigator (JM) wrote the code for these calculations.

\section{Levels of Evidence and Strength of Recommendation}

The Oxford Centre for Evidence-based Medicine Levels of Evidence taxonomy, developed by Phillips et al, ${ }^{43}$ was used as to characterize the quality, quantity, and consistency of the included studies. The level of evidence for the included studies and strength of recommendation for the use of neural gliding for the treatment of CTS was determined using these algorithms.

\section{Evidence Synthesis}

\section{Study Selection}

The literature search resulted in 20 relevant studies. Six studies ${ }^{28,29,41,44-46}$ met the predetermined criteria and were included in this systematic review. The remaining 14 studies were excluded. Reasons for rejection included literature review, ${ }^{47-51}$ clinical commentary, ${ }^{52-55}$ previous systematic review, ${ }^{25-27}$ no comparison group,${ }^{56}$ cadaver studies, ${ }^{24,57,58}$ and other studies that did not address neural gliding techniques for the treatment of carpal tunnel syndrome. ${ }^{59-62}$

\section{Homogeneity}

All groups of the included studies were similar at baseline. All patients were being treated for CTS. Mean ages of the groups were similar (neural gliding groups $=48.7$ years, comparison treatment $=48.8$ years). Duration of symptoms was not clearly defined; some studies reported mean duration across all subjects, some studies reported ranges of symptom duration. Symptom duration across all studies ranged from $<6$ months to $>2$ years. All studies used previously described techniques for mobilizing the median nerve; four studies ${ }^{28,41,44,45}$ used similar techniques as previously described, ${ }^{46,63}$ and one study ${ }^{29}$ used the pattern of the Upper Limb Tension Test 2 for mobilization of the median nerve. There was variation across studies in regards to neural gliding exercise prescription, follow-up periods, and comparison (control) group courses of treatment. Please refer to Table 1 for subject demographics of included studies.

The interventions performed on the control groups were not standardized across studies. One study ${ }^{29}$ used a true control and also a comparison was made to a carpal mobilization treatment group. Each was compared separately to neural gliding in this systematic review. Baysal et $\mathrm{al}^{44}$ utilized splint + ultrasound as the control group for comparison to neural gliding. Three studies ${ }^{28,41,45}$ used a splint only as the control group. Brininger et $\mathrm{al}^{41}$ used two different types of splint; each of these was compared to neural gliding for this systematic review. 


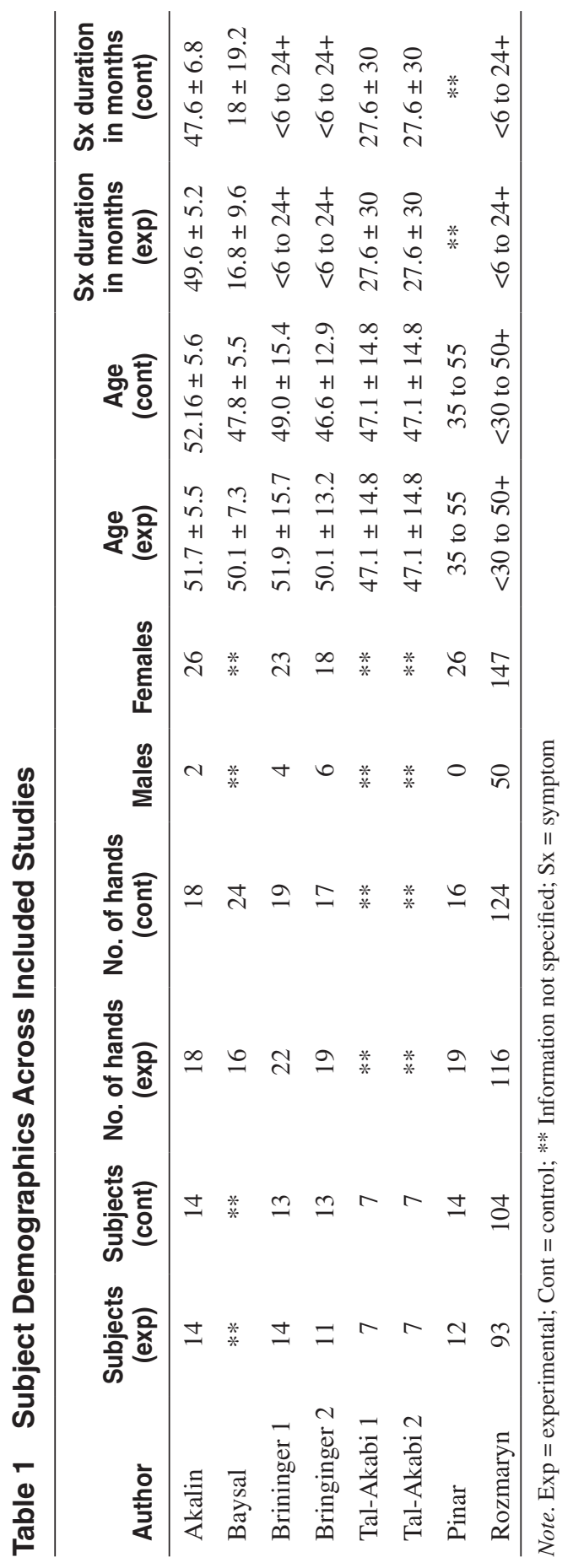




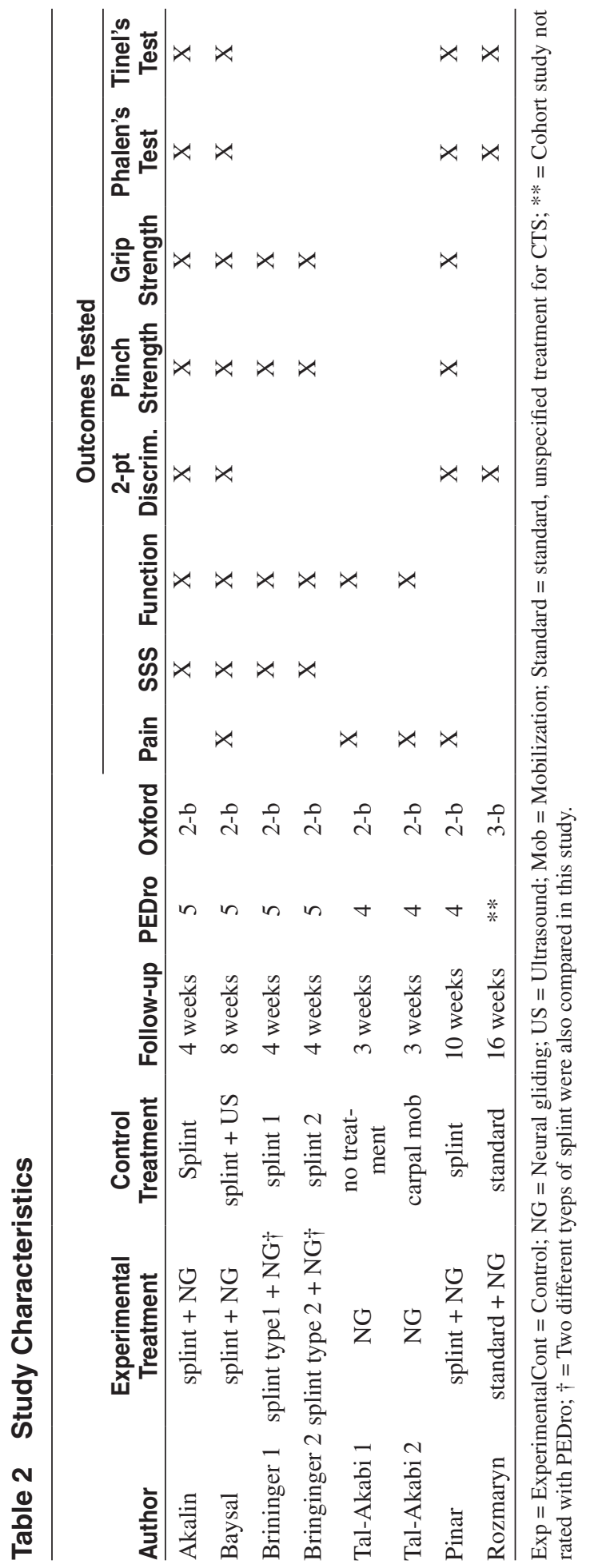


All studies used at least one of the eight previously mentioned clinical outcomes. For all outcomes, except one (self-reported function in Tal-Akabi and Rushton ${ }^{29}$ ), similar assessment tools were administered across studies. In addition, all studies included at least one follow-up outcome measurement; however, there was a relatively short range of follow-up time periods (4 to 10 weeks). Although the outcome measures were similar across studies, only two studies incorporated all eight measures. In addition, the treatment of the control groups was not uniform across studies. The heterogeneity of the interventions utilized for the control groups, in addition to the limited number of comparable outcomes across studies, were the major factors in the decision to perform a systematic review, rather than meta-analysis. Please refer to Table 2 for individual study characteristics.

\section{Methodological Quality and Study Characteristics}

Each investigator independently scored the remaining studies utilizing Physiological Evidence Database (PEDro) rating scale. There was total agreement for PEDro scores of all reviewed studies. The highest PEDro rating was 5/10 and with an average of 4.6 among the included studies. Please refer to Table 2 for individual PEDro scores for each included study.

\section{Data Synthesis}

Self-Reported Outcomes (Pain, SSS, FSS) and Two-Point Discrimination. Figure 1 illustrates the effect sizes and $95 \%$ confidence intervals for self-reported outcomes and two-point discrimination. For the outcome of pain, three ${ }^{29,45}$ out of four ${ }^{29,44,45}$ comparisons demonstrated weak to strong (effect sizes ranged from -.3 to -2.1 ) effect sizes that favored neural gliding. Only one comparison ${ }^{29}$ (Tal-Akabi: neural gliding vs. true control), which was favorable to neural gliding, also demonstrated a $95 \%$ confidence interval that did not encompass zero. For reported symptom severity, only one ${ }^{28}$ out of four ${ }^{28,41,44}$ comparisons demonstrated moderate, but favorable, effects for neural gliding, with wide $95 \%$ confidence intervals that encompassed zero (effect sizes ranged from -.4 to .1). Four studies ${ }^{28,29,41,44}$ made six comparisons that included the outcome of self-reported function during patient assessment. Five $^{28,29,41,44}$ of these six demonstrated weak to strong effects that favored neural gliding (effect sizes for all six comparisons ranged from -1.3 to .3). Of these, two demonstrated moderate to strong effects and $95 \%$ confidence intervals that did not encompass zero. For two-point discrimination, one ${ }^{28}$ of two studies ${ }^{28,44}$ demonstrated moderate effect sizes that favored neural gliding; however, the $95 \%$ confidence interval encompassed zero.

Strength (Grip Strength and Pinch Strength). The effect sizes and 95\% confidence intervals for the outcomes of grip strength and pinch strength are shown in Figure 2. The specific testing position was not described in three $28,44,45$ of the four ${ }^{28,41,44,45}$ studies that included pinch strength. In the study by Brininger et al, ${ }^{41}$ three different positions were specified and tested for pinch strength. The effect sizes and confidence intervals for all three positions are represented in Figure 2. For the nine comparisons of pinch strength in four studies ${ }^{28,41,44,45}$ that utilized pinch strength as an outcome, all demonstrated weak to moderate effects that favored neural gliding; however, only one ${ }^{41}$ (Brininger: neural gliding vs. splint 2 ) demonstrated a $95 \%$ confidence interval that did not encompass zero. For grip strength, 


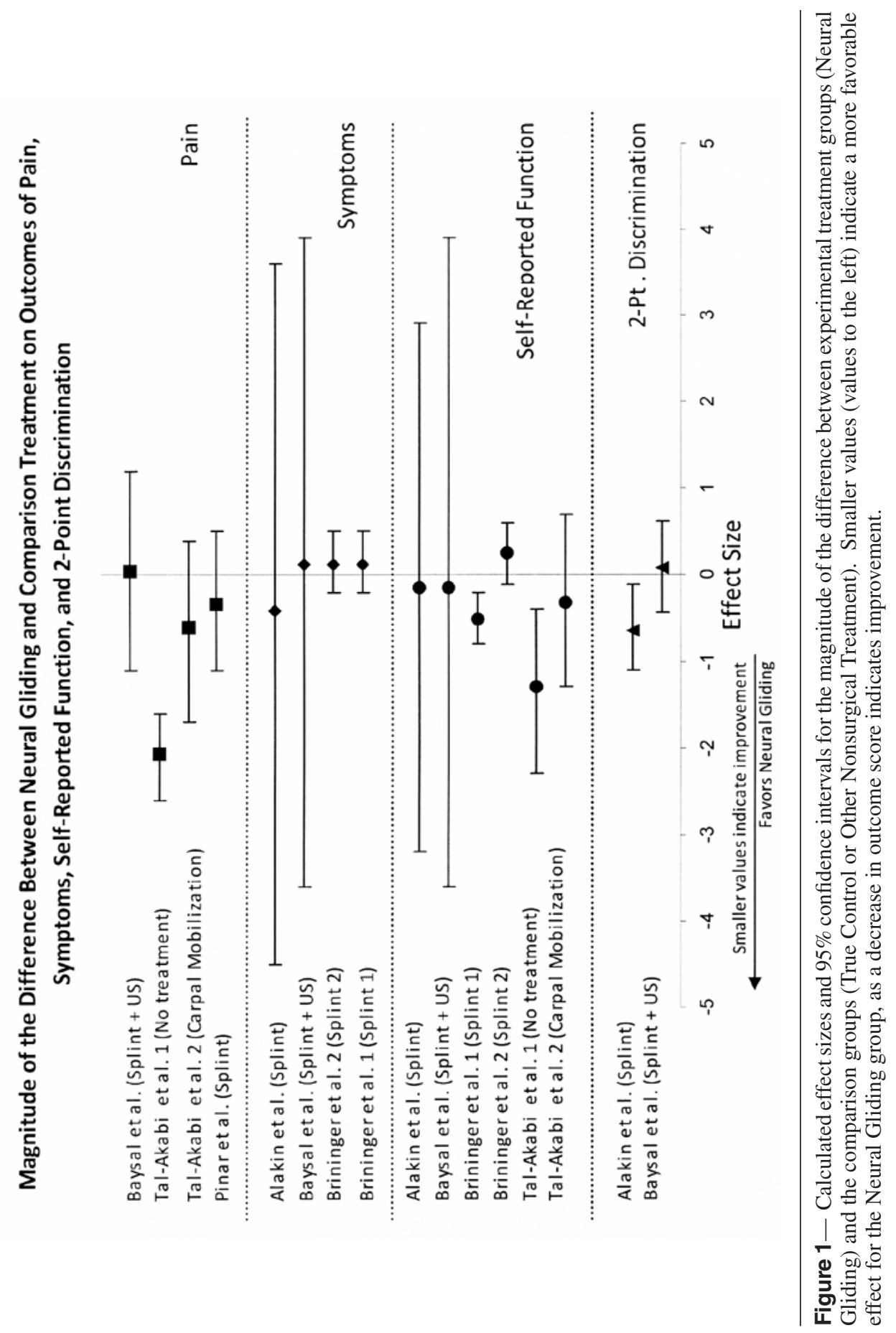




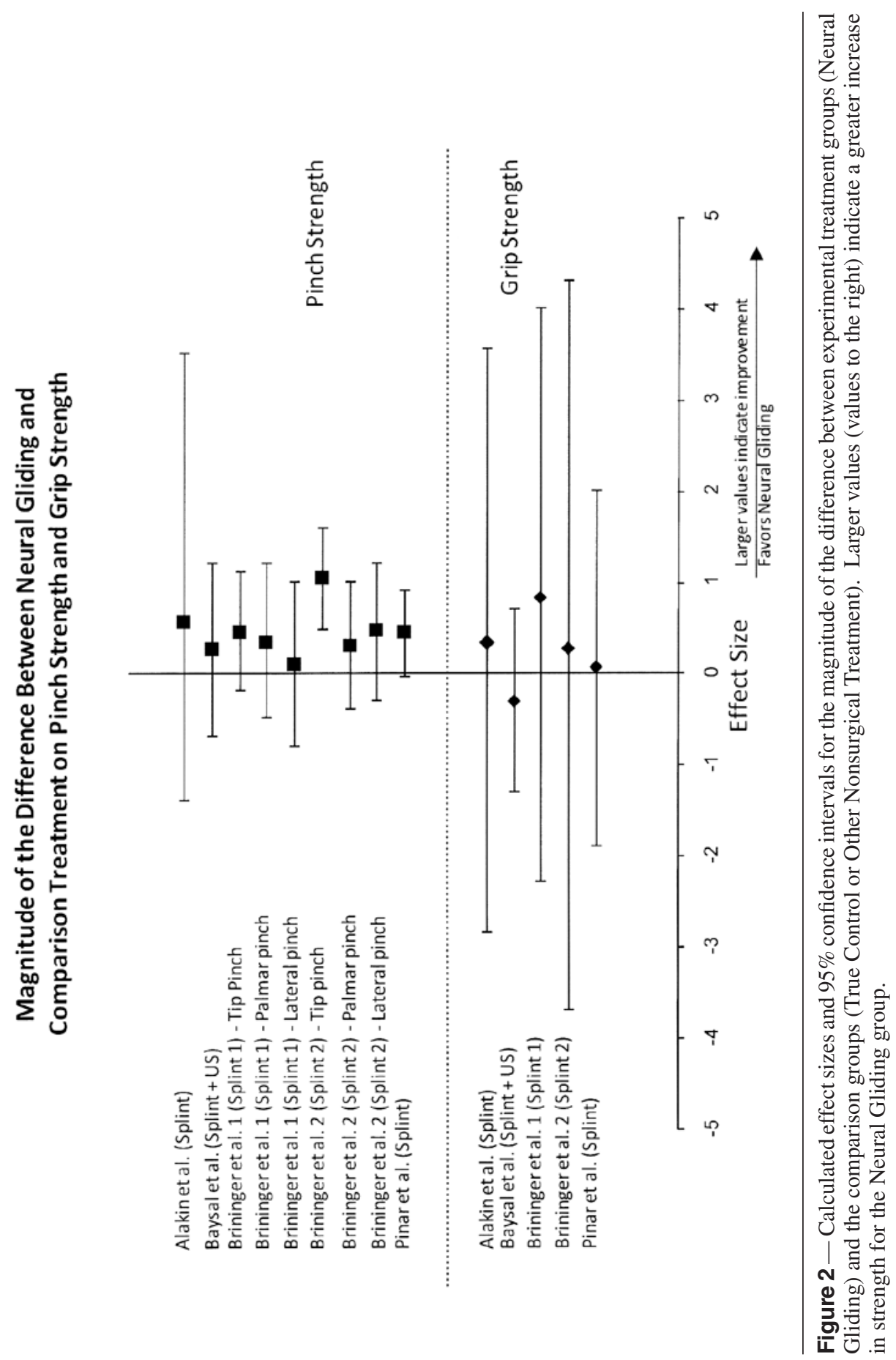


four out of five comparisons in four studies ${ }^{28,41,44,45}$ demonstrated weak to moderate effects that favored neural gliding; however, all demonstrate wide $95 \%$ confidence intervals that encompassed zero.

Clinical Exam (Phalen's Test and Tinel's Test). Figure 3 depicts the relative risk of success (ie, an unremarkable result on the clinical special test after treatment) and 95\% confidence intervals for Phalen's test and Tinel's test. For the studies ${ }^{28,44-46}$ that used Phalen's test as an outcome, relative risk of success was demonstrated as favorable toward neural gliding groups in three ${ }^{28,45,46}$ of four comparisons; however, all 95\% confidence intervals were wide and encompassed zero (relative risk ranged from .8 to 1.9; risk difference ranged from -.2 to .2). For Tinel's test, two ${ }^{28,44}$ out of four ${ }^{28,44-46}$ comparisons demonstrated risk ratios that favored neural gliding; however, these also demonstrated wide confidence intervals that encompassed zero (relative risk ranged from .8 to 1.2 ; risk difference ranged from -.01 to .1).

\section{Levels of Evidence and Strength of Recommendation}

A summarization of evidence of these lower quality clinical trials and a cohort study is limited to level 2-b and 3-b Evidence. The Strength of Recommendation for the use of neural gliding is grade B. We base this recommendation on the poor methodological quality of the included studies and inconsistencies in the effects of neural gliding over alternative nonsurgical treatment.

\section{Discussion}

The purpose of this systematic review was to determine the efficacy of neural gliding for the treatment of CTS. Although previous systematic reviews have been performed on this topic, we felt justified to conduct a more comprehensive analysis that included the most current available evidence. New studies ${ }^{41,44,45}$ were included in the current systematic review that were not included in any of the three previous reviews. In addition, we also performed an objective comparison of the studies by calculating effect sizes and risk ratios of the results, resulting in more unbiased conclusions in the current systematic review. This was not done for the systematic reviews by Muller et $\mathrm{al}^{26}$ and Goodyear-Smith et al. ${ }^{25}$ Most importantly, we believe that we have identified areas that may have obscured the true efficacy of neural gliding in these previous clinical trials and make recommendations for further research to help identify better application for neural gliding in the treatment of CTS.

Results of the current systematic review demonstrate weak to strong effect of neural gliding exercises with benefits seen across all outcome measures. Comparisons across studies suggest a benefit of neural gliding exercises for CTS; however, the efficacy remains unclear due to $95 \%$ confidence intervals that encompass zero across all outcome measures. Calculated risk ratios for Phalen's test were biased slightly, but not definitively, toward favoring neural gliding. Risk ratios for Tinel's test did not demonstrate any favorable trend toward treatment or no treatment. The contribution made by neural gliding exercises to a combination treatment approach is unknown. However, based on the trend toward improved outcomes with the inclusion of neural gliding, we conclude that neural gliding is better than no treatment. 


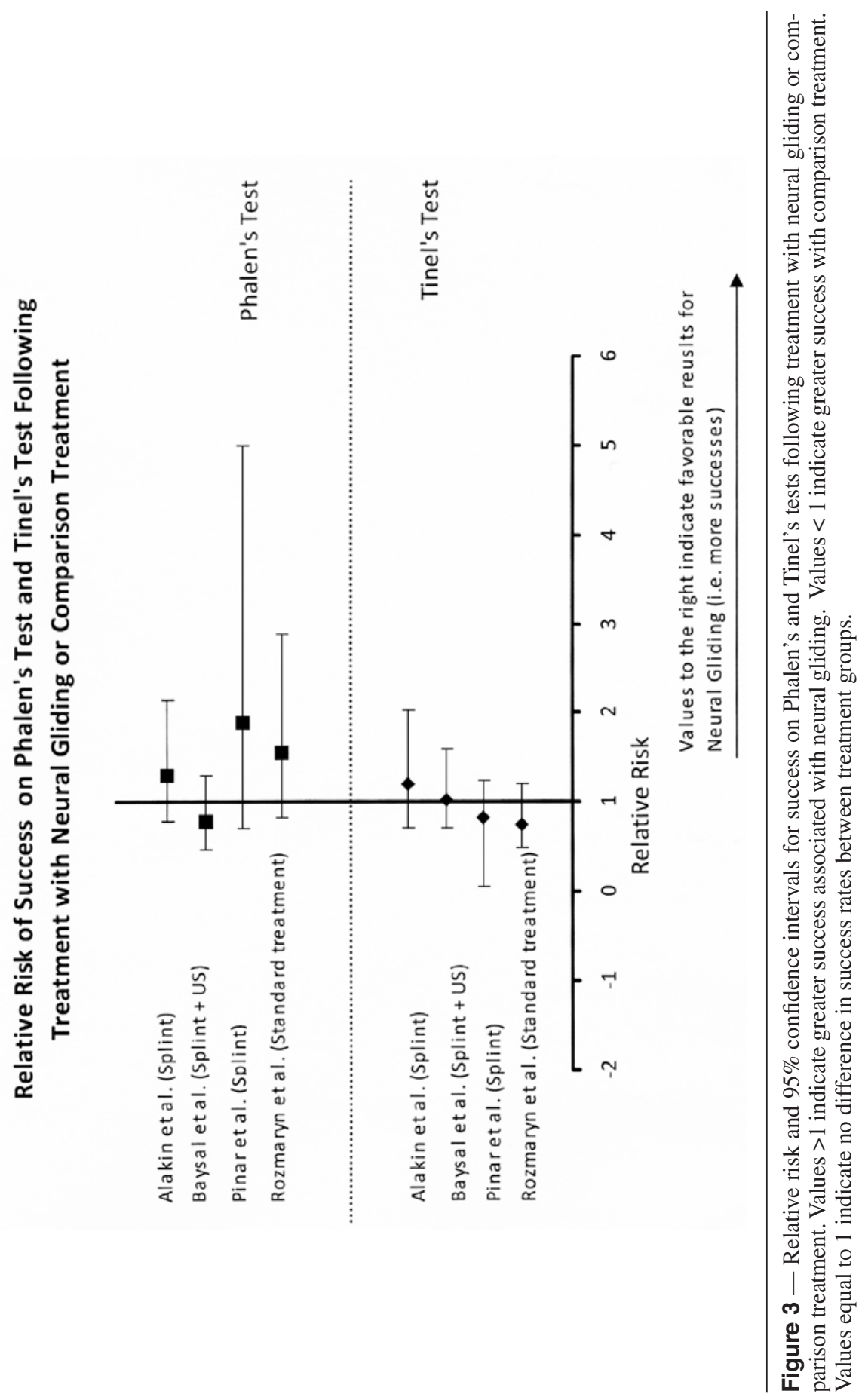


Three previous systematic reviews ${ }^{25-27}$ that included neural gliding were identified. O'Connor et $\mathrm{al}^{27}$ and Muller et $\mathrm{al}^{26}$ each performed systematic reviews that included the same two studies ${ }^{28,29}$ and each made opposing conclusions based on study results.

O'Connor et $\mathrm{al}^{27}$ concluded no significant effect of neural gliding for the treatment of CTS. In contrast, Muller et $\mathrm{al}^{26}$ included the same two studies ${ }^{28,29}$ and concluded that a neural mobilization was more effective in relieving pain than no treatment. Muller et $\mathrm{al}^{26}$ explained this discrepancy by post-hoc analyses of the data to identify specific group differences that were not reported by Tal-Akabi and Rushton. ${ }^{29}$ From these post-hoc analyses, it was identified that the neural gliding group did perform significantly better than the no treatment group. These post-hoc analyses to identify group differences were not performed by O'Connor et al, ${ }^{27}$ which explains the discrepancy in the conclusions for these systematic reviews. This particular review provided good documentation of research study quality to determine level of evidence; however, it did not provide clinically meaningful standardized values to compare across studies. The third review ${ }^{25}$ only included one study ${ }^{46}$ that addressed neural gliding exercises and found no significant change in Phalen's or Tinel's tests results, however, and concluded that neural gliding may be an effective nonsurgical treatment for CTS.

The benefit of neural gliding may be best identified within a specific subpopulation of CTS patients. It is possible that neural gliding may be more effective in a population with less advanced CTS. Of the studies reviewed, only two ${ }^{41,45}$ specifically stated that severe CTS was an exclusion criterion. Their exclusion of the worst cases of CTS into the trials was likely done to avoid a wash out effect of any possible benefits of neural gliding. This decision captures the clinical reasoning that conservative treatment is directed at patients with mild to moderate symptoms in order to delay or prevent disease progression and subsequent surgical interventions. For patients with severe CTS, surgical intervention may be the only effective treatment option to alleviate compression of the median nerve and reduce disease progression. To identify the population that may respond optimally to this conservative treatment intervention, future studies should stratify the patient population by severity or progression of the disease.

Identifying the specific pathogenesis of CTS may be important in determining the true efficacy of neural gliding exercises. The following three etiologies are suggested to cause CTS by eliciting compression of the neurovascular system as it passes through the carpal tunnel: (1) ischemia, (2) a decrease in longitudinal excursion of the median nerve, and (3) mechanical compression or injury to involved carpal structures.

CTS caused by ischemia may be positively affected by treatment with neural gliding exercises. Nerves are sensitive to prolonged ischemic states due to the high demand of circulating oxygen. ${ }^{64}$ Neural gliding exercises may relieve ischemic pain by contributing to the delivery of oxygenated blood to the median nerve at its distal site within the wrist and hand.

In a recent study, ${ }^{23}$ Hough et al identified, with the use of Doppler ultrasound, that limited longitudinal excursion of the median nerve exists in patients with CTS. There is also evidence that median nerve excursion can be influenced by neural gliding techniques, as demonstrated in a cadaveric study. ${ }^{24}$ The results of these studies lend credibility to the use of movement-based interventions such as neural 
gliding exercises. Moreover, the use of Doppler ultrasound may be clinically useful in identifying patients who may respond favorably to neural gliding.

Finally, the etiology of mechanical compression should be examined. The hypothesis that biomechanical factors cause CTS is well accepted. Factors include intrinsic characteristics such as bony and soft tissue structural variations within the carpal tunnel as well as extrinsic factors such as prolonged, awkward postures of extreme wrist angles. Clinical reasoning supported rest and splinting as an intervention for this etiology, as opposed to neural gliding exercises; however, it is possible that neural gliding exercises may also benefit patients with mechanical compression of the median nerve.

Improvements in diagnostic medicine will lead to better and more parsimonious rehabilitation programs. Clinicians will be able to more judiciously prescribe neural exercises to patients who are likely to benefit based on etiology, rather than prescribing the exercises based only on symptoms. This would be a significant contribution to evidence based rehabilitation science; however, at this current time, more research is needed to identify better clinical prediction rules and to more closely examine pathogenesis of CTS. The use of neural gliding in relation to each of the above etiologies is discussed below.

The incorporation of neural gliding exercises should also be considered from a cost-benefit perspective. The monetary and temporal cost of performing these exercises is minimal. Incorporating these exercises into a home exercise program is cost-effective and may improve a patient's symptoms and function. In addition, the practice of incorporating neural gliding exercises, in combination with other interventions, may delay the need for surgery. ${ }^{25}$

\section{Recommendations for Future Research}

The most current and best available evidence in regard to neural gliding exercises for treatment of CTS comes from few uncontrolled, randomized clinical trials, incomplete systematic reviews, and anecdotal clinical evidence. Future recommendations for research include well-controlled randomized clinical trails, longer follow-up periods, and high quality research on combination treatment. Blinding of patients, clinicians, and administrators is often difficult in physical rehabilitation studies, and investigators will have to be more creative and improve methods of blinding within studies in order to reduce potential study bias. The studies included in this systematic review reported outcomes for a relatively short period of time after treatment. How long does this treatment last, and can it eliminate the need for surgical intervention on a patient, are questions that need to be answered. It is likely that neural gliding exercises will be included in an effective rehabilitation program, designed to improve symptoms and function in a specific CTS population. The use of neural gliding exercises as a prophylactic measure for athletes at a higher risk for CTS should also be examined. More high-quality research should be directed at identifying the best combinations of treatments and identifying the patient populations that will most benefit from these interventions.

In addition, more uniform control and reporting of the protocols followed by both the neural gliding and comparison groups are needed. For the neural gliding group, well-defined treatment protocols should be used by all subjects throughout the study. For the comparison groups, more control standardized procedures are 
needed during which the subjects are not receiving a treatment that may confound results. Although it is ethically difficult to deny standard treatment to CTS patients, more uniform control treatment will allow investigators to identify the best combination of treatments for CTS. We recommend splinting as an ethical and traditional form of control treatment for CTS that has also been determined to be efficacious. ${ }^{65,66}$

\section{Conclusions}

It appears that neural gliding exercise may aid in the reduction of symptoms and improvement in function in patients with CTS; however, the relatively weak response in the sample incorporated in our analysis does not provide sufficient reason to recommend these exercises as the best available nonsurgical treatment of CTS.

While the efficacy of neural gliding techniques for the treatment of CTS is not clear, trends toward pain and symptom reduction, improved sensation, and improved function and strength, combined with the low monetary and temporal cost of the treatment, make this treatment a reasonable option for clinicians in treating individuals with this disorder.

\section{Practice Recommendations}

- Neural gliding exercises may provide short-term improvement in pain, function, and strength.

- It is likely that patients with carpal tunnel syndrome (CTS) will benefit from the addition of neural gliding to standard treatment for CTS.

- The efficacy of neural gliding for treatment of less severe cases is unknown. Future research should incorporate the stratification of patients by severity.

\section{References}

1. Moscony AMB. Common peripheral nerve problems. In: Cooper C, ed. Fundamentals of Hand Therapy: Clinical Reasoning and Treatment Guidelines for Common Diagnoses of the Upper Extremity. Philadelphia: Mosby Elsevier; 2007:201-250.

2. Falkiner S, Myers S. When exactly can carpal tunnel syndrome be considered workrelated? ANZ J Surg. 2002;72:204-209.

3. U.S. Department of Labor. Occupational Injuries and Illnesses: Counts, Rates, and Characteristics. Bulletin 2538. Washington, DC: U.S. Bureau of Labor Statistics; 2001.

4. National Institute of Neurological Disorders and Stroke. Carpal Tunnel Syndrome Fact Sheet. Available at: http://www.ninds.nih.gov/disorders/carpal_tunnel/detail_carpal_ tunnel.htm?css=print. Accessed April 25, 2008.

5. Nuber GW, Assenmacher J, Bowen MK. Neurovascular problems in the forearm, wrist, and hand. Clin Sports Med. 1998;17:585-610.

6. Lorei MP, Hershman EB. Peripheral nerve injuries in athletes. Treatment and prevention. Sports Med. 1993;16:130-147.

7. Wood MB, Dobyns JH. Sports-related extraarticular wrist syndromes. Clin Orthop Relat Res. 1986:93-102.

8. Krivickas LS, Wilbourn AJ. Peripheral nerve injuries in athletes: a case series of over 200 injuries. Semin Neurol. 2000;20:225-232. 
9. Toth C, McNeil S, Feasby T. Peripheral nervous system injuries in sport and recreation: a systematic review. Sports Med. 2005;35:717-738.

10. Seradge H, Jia YC, Owens W. In vivo measurement of carpal tunnel pressure in the functioning hand. J Hand Surg [Am]. 1995;20:855-859.

11. Akuthota V, Plastaras C, Lindberg K, Tobey J, Press J, Garvan C. The effect of longdistance bicycling on ulnar and median nerves: an electrophysiologic evaluation of cyclist palsy. Am J Sports Med. 2005;33:1224-1230.

12. Parziale JR, Mallon WJ. Golf injuries and rehabilitation. Phys Med Rehabil Clin NAm. 2006;17:589-607.

13. Holtzhausen LM, Noakes TD. Elbow, forearm, wrist, and hand injuries among sport rock climbers. Clin J Sport Med. 1996;6:196-203.

14. Burnham RS, Steadward RD. Upper extremity peripheral nerve entrapments among wheelchair athletes: prevalence, location, and risk factors. Arch Phys Med Rehabil. 1994;75:519-524.

15. Werner RA, Andary M. Carpal tunnel syndrome: pathophysiology and clinical neurophysiology. Neurophysiology Clinics. 2002;113:1373-1381.

16. von Schroeder HP, Botte MJ. Carpal tunnel syndrome. Hand Clin. 1996;12:643-655.

17. McLellan DL, Swash M. Longitudinal sliding of the median nerve during movements of the upper limb. J Neurol Neurosurg Psychiatry. 1976;39:566-570.

18. Hunter JM. Recurrent carpal tunnel syndrome, epineural fibrous fixation, and traction neuropathy. Hand Clin. 1991;7:491-504.

19. Valls-Sole J, Alvarez R, Nunez M. Limited longitudinal sliding of the median nerve in patients with carpal tunnel syndrome. Muscle Nerve. 1995;18:761-767.

20. Bay BK, Sharkey NA, Szabo RM. Displacement and strain of the median nerve at the wrist. J Hand Surg [Am]. 1997;22:621-627.

21. Szabo RM, Bay BK, Sharkey NA, Gaut C. Median nerve displacement through the carpal canal. J Hand Surg [Am]. 1994;19:901-906.

22. Wright TW, Glowczewskie F, Wheeler D, Miller G, Cowin D. Excursion and strain of the median nerve. J Bone Joint Surg Am. 1996;78:1897-1903.

23. Hough AD, Moore AP, Jones MP. Reduced longitudinal excursion of the median nerve in carpal tunnel syndrome. Arch Phys Med Rehabil. 2007;88:569-576.

24. Ettema AM, Zhao C, Amadio PC, O'Byrne MM, An KN. Gliding characteristics of flexor tendon and tenosynovium in carpal tunnel syndrome: a pilot study. Clin Anat. 2007;20:292-299.

25. Goodyear-Smith F, Arroll B. What can family physicians offer patients with carpal tunnel syndrome other than surgery? A systematic review of nonsurgical management. Ann Fam Med. 2004;2:267-273.

26. Muller M, Tsui D, Schnurr R, Biddulph-Deisroth L, Hard J, MacDermid JC. Effectiveness of hand therapy interventions in primary management of carpal tunnel syndrome: a systematic review. $J$ Hand Ther. 2004;17:210-228.

27. O'Connor D, Marshall S, Massy-Westropp N. Non-surgical treatment (other than steroid injection) for carpal tunnel syndrome. Cochrane Database Syst Rev. 2003:CD003219.

28. Akalin E, El O, Peker O, Senocak O, Tamci S, Gülbahar S, Cakmur R, Oncel S. Treatment of carpal tunnel syndrome with nerve and tendon gliding exercises. Am J Phys Med Rehabil. 2002;81:108-113.

29. Tal-Akabi A, Rushton A. An investigation to compare the effectiveness of carpal bone mobilisation and neurodynamic mobilisation as methods of treatment for carpal tunnel syndrome. Man Ther. 2000;5:214-222.

30. Collins SL, Moore RA, McQuay HJ. The visual analogue pain intensity scale: what is moderate pain in millimetres? Pain. 1997;72:95-97.

31. Ferraz MB, Quaresma MR, Aquino LR, Atra E, Tugwell P, Goldsmith CH. Reliability of pain scales in the assessment of literate and illiterate patients with rheumatoid arthritis. J Rheumatol. 1990;17:1022-1024. 
32. Harms-Ringdahl K, Carlsson AM, Ekholm J, Raustorp A, Svensson T, Toresson HG. Pain assessment with different intensity scales in response to loading of joint structures. Pain. 1986;27:401-411.

33. Price DD, McGrath PA, Rafii A, Buckingham B. The validation of visual analogue scales as ratio scale measures for chronic and experimental pain. Pain. 1983;17:45-56.

34. Mattacola CG, Perrin DH, Gansneder BM, Allen JD, Mickey CA. A comparison of visual analog and graphic rating scales for assessing pain following delayed onset muscle soreness. J Sport Rehabil. 1997;6:38-46.

35. Levine DW, Simmons BP, Koris MJ, Daltroy LH, Hohl GG, Fossel AH, Katz JN. A self-administered questionnaire for the assessment of severity of symptoms and functional status in carpal tunnel syndrome. J Bone Joint Surg Am. 1993;75:1585-1592.

36. Hoppenfeld S. Physical Examination of the Spine and Extremities. Norwalk, CT: Appleton \& Lange; 1976.

37. Starkey C, Ryan J. Evaluation of Orthopedic and Athletic Injuries. Philadelphia: F.A. Davis Company; 2002.

38. Benaglia PG, Franchignoni F, Ferriero G, Zebellin G, Sartorio F. Giornale italiano di medicina del lavoro ed ergonomia. [Reliability and validity of the analysis of hand grip and pinch force in isometric and isokinetic conditions]. G Ital Med Lav Ergon. 1999;21:20-24.

39. Watanabe T, Owashi K, Kanauchi Y, Mura N, Takahara M, Ogino T. The short-term reliability of grip strength measurement and the effects of posture and grip span. $J$ Hand Surg. 2005;30:603-609.

40. Mathiowetz V, Weber K, Volland G, Kashman N. Reliability and validity of grip and pinch strength evaluations. J Hand Surg. 1984;9:222-226.

41. Brininger TL, Rogers JC, Holm MB, Baker NA, Li ZM, Goitz RJ. Efficacy of a fabricated customized splint and tendon and nerve gliding exercises for the treatment of carpal tunnel syndrome: a randomized controlled trial. Arch Phys Med Rehabil. 2007;88:1429-1435.

42. Cohen J. Statistical Power Analysis for the Behavioral Sciences. 2nd ed. Hillsdale, NJ: Lawrence Erlbaum Associates, Inc.; 1988.

43. Phillips B, Ball C, Sackett D, Badenoch D, Straus S, Haynes B, Dawes M. Oxford Centre for Evidence-based Medicine Levels of Evidence. Available on-line. Accessed April, 2008.

44. Baysal O, Altay Z, Ozcan C, Ertem K, Yologlu S, Kayhan A. Comparison of three conservative treatment protocols in carpal tunnel syndrome. Int $J$ Clin Pract. 2006;60:820-828.

45. Pinar L, Enhos A, Ada S, Gungor N. Can we use nerve gliding exercises in women with carpal tunnel syndrome? Adv Ther. 2005;22:467-475.

46. Rozmaryn LM, Dovelle S, Rothman ER, Gorman K, Olvey KM, Bartko JJ. Nerve and tendon gliding exercises and the conservative management of carpal tunnel syndrome. J Hand Ther. 1998;11:171-179.

47. Michlovitz SL. Conservative interventions for carpal tunnel syndrome. J Orthopaedic Sports Phys Ther. 2004;34:589-600.

48. Burke FD, Ellis J, McKenna H, Bradley MJ. Primary care management of carpal tunnel syndrome. Postgrad Med J. 2003;79:433-437.

49. Kietrys DM. Neural mobilization: an appraisal of the evidence regarding validity and efficacy. Orthopaedic Phys Ther Practice. 2003;15:18-20.

50. Kostopoulos D. Treatment of carpal tunnel syndrome: a review of the non-surgical approaches with emphasis in neural mobilization. J Bodywork Movement Ther. 2004;8:2-8.

51. MacDermid JC, Doherty T. Clinical and electrodiagnostic testing of carpal tunnel syndrome: a narrative review. J Orthop Sports Phys Ther. 2004;34:565-588. 
52. Foxman I, Burgel BJ. Musician health and safety: Preventing playing-related musculoskeletal disorders. Aaohn J. 2006;54:309-316.

53. Walsh MT. Upper limb neural tension testing and mobilization: fact, fiction, and a practical approach. J Hand Ther. 2005;18:241-258.

54. Schroder JA. Manual therapy and neural mobilization: our approach and personal observations. Orthopaedic Phys Ther Practice. 2004;16:23-27.

55. Seradge H. Carpal tunnel syndrome: surgical and nonsurgical treatment. J Hand Surg [Am]. 1994;19:704.

56. Seradge H, Parker W, Baer C, Mayfield K, Schall L. Conservative treatment of carpal tunnel syndrome: an outcome study of adjunct exercises. J Okla State Med Assoc. 2002;95:7-14.

57. Coppieters MW, Butler DS. Do 'sliders' slide and 'tensioners' tension? An analysis of neurodynamic techniques and considerations regarding their application. Man Ther. 2007; epub.

58. Coppieters MW, Alshami AM. Longitudinal excursion and strain in the median nerve during novel nerve gliding exercises for carpal tunnel syndrome. J Orthop Res. 2007;25:972-980.

59. Ettema AM, Amadio PC, Cha SS, Harrington JR, Harris AM, Offord KP. Surgery versus conservative therapy in carpal tunnel syndrome in people aged 70 years and older. Plast Reconstr Surg. 2006;118:947-958; discussion 959-960.

60. Sheon RP. Peripheral nerve entrapment, occupation-related syndromes, and sports injuries. Curr Opin Rheumatol. 1992;4:219-225.

61. Harter BT, Jr., McKiernan JE, Jr., Kirzinger SS, Archer FW, Peters CK, Harter KC. Carpal tunnel syndrome: surgical and nonsurgical treatment. J Hand Surg [Am]. 1993;18:734-739.

62. Gerritsen AA, de Krom MC, Struijs MA, Scholten RJ, de Vet HC, Bouter LM. Conservative treatment options for carpal tunnel syndrome: a systematic review of randomised controlled trials. J Neuro. 2002;249:272-280.

63. Totten PA, Hunter JM. Therapeutic techniques to enhance nerve gliding in thoracic outlet syndrome and carpal tunnel syndrome. Hand Clin. 1991;7:505-520.

64. Butler DS. Mobilisation of the Nervous System. 1st ed. Melbourne: Churchill Livingstone; 1991.

65. Manente G, Torrieri F, Di Blasio F, Staniscia T, Romano F, Uncini A. An innovative hand brace for carpal tunnel syndrome: a randomized controlled trial. Muscle Nerve. 2001;24:1020-1025.

66. Walker WC, Metzler M, Cifu DX, Swartz Z. Neutral wrist splinting in carpal tunnel syndrome: a comparison of night-only versus full-time wear instructions. Arch Phys Med Rehabil. 2000;81:424-429. 